\title{
From Microstructures of Tablets and Granules to Their Dissolution Behaviour
}

\author{
Xiaodong Jia ${ }^{1,2,3}$ and Richard A. Williams ${ }^{1}$
}

e-mail:X.Jia@leeds.ac.uk

\section{Introduction}

D issolution testing is now an established and standardised method for measuring the performance of drug products (1). It allows results for different batches of the same product, similar products from different suppliers, or tests from different labs to be compared. It is, therefore, a useful tool for quality control and for formulation development, so much so that the FDA has made it a regulatory requirement for approval of new drugs. However, being an empirical method, it does have some drawbacks for formulation development. For example, existing empirical data or formula may be of little help for every new formulation, and a new series of tests may have to be performed. For every dissolution test, the sample product must have already been physically made. Both significantly add to the time and cost of taking a new drug to market. A theoretical model, on the other hand, can help to explain experimental observations and to predict the likely outcome of a new formulation at the design stage, thereby reducing the number of physical tests that have to be conducted and the total cost and time of drug development.

This article introduces to the dissolution testing community a new computer modelling approach and demonstrates its capability and potential to fulfil the above role through hypothetical yet illustrative examples. It is called DigiPac ${ }^{T M}$ Applications Suite ${ }^{*}$, based on a patented digital approach. It differs from existing models in two important ways. First, it is a mesoscale digital approach. In comparison, most existing models take a macroscopic vector-based approach. Secondly, and more importantly, it handles with ease real particle shapes, rather than some idealised geometrical models.

Being a particle-level, or mesoscale, numerical model, it predicts the influence of particle size and shape distributions on the microstructure of granules and of tablets, and from the microstructure, the dissolution behaviour. The software implementation of the DigiPac approach includes modules for particle packing, flow calculation, and dissolution simulation. For tabletting, DigiPac links fundamental properties of excipient and incipient particles, through the initial packing and ultimately compaction, to the structure of the tablets. For dissolution, the starting point is a digitised microstructure of mixed components. The digital structure is either a simulated one using DigiPac packing module or a real one obtained using X-ray microtomography (XMT). Diffusion-convection equation is solved for each component using a finite difference scheme with the classic NoyesWhitney equation acting as the boundary condition at solid-liquid interfaces. The flow field, which is another input for dissolution simulations, is either calculated using a numerical technique called Lattice Boltzmann Method (LBM) or measured experimentally (e.g., in a dissolution test apparatus).

Since DigiPac, XMT, and LBM are integral parts of the dissolution model and each is a relatively new technique (none being commercially available for more than five years), they are briefly described first, before the dissolution model itself is introduced and demonstrated.

\section{Digitisation of Particle Shape and Tablet Structure}

An overview of the current technology in acquiring shapes of real particles and microstructures of tablets is given elsewhere (2). Essentially, there is, as yet, no dedicated equipment for real-time digitisation of particles in 3D, but machines based on different measuring principles and capable of providing 3D information of fine particles are commercially available and increasingly being used as such. One such example is X-ray microtomography (XMT) scanners such as SkyScan 1172 and Phoenix Nanotom.

Principles of computerised tomographic imaging for medical use are described by Kak and Slaney (3). Essentially, hundreds of X-ray images taken from known angles are collected and combined by following a mathematical procedure, called a reconstruction algorithm, to give a series of cross-sectional slices, known as reconstructed images or tomograms, which can then be stacked to form a 3D digital structure of the sample. The X-ray images, and hence the reconstructed tomograms, map the spatial distribution of relative mass density within the sample. Knowing what components the sample contains and their mass densities allows one to distinguish one component from another. Applying thresholds to retain pixels of a certain range allows one to extract a particular component and study its spatial distribution in the sample. Provided particles of this particular component are large enough (e.g., at least 5 pixels

\footnotetext{
'Institute of Particle Science and Engineering, School of Process, Environmental and Materials Engineering, University of Leeds, Leeds LS2 9JT, UK

* DigiPac ${ }^{\mathrm{TM}}$ is a product of Structure Vision Ltd.
}

${ }_{2}^{2}$ Structure Vision Ltd, Leeds Innovation Centre, 103 Clarendon Road, Leeds LS2 9DF, UK

${ }^{3}$ Corresponding author. 


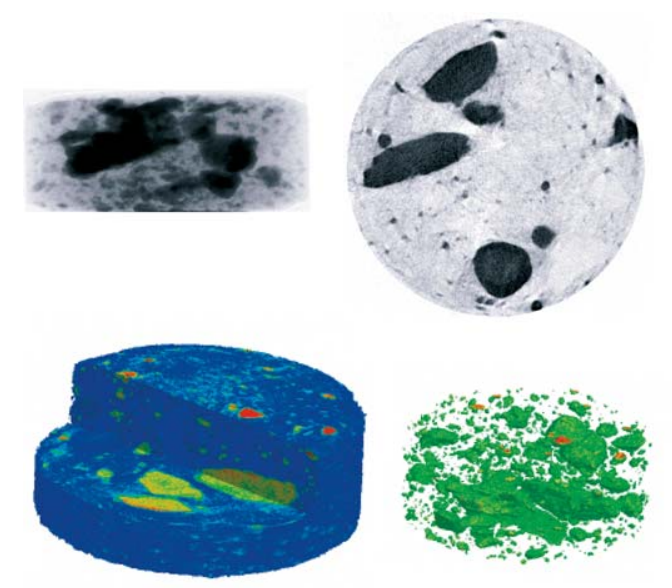

(a)

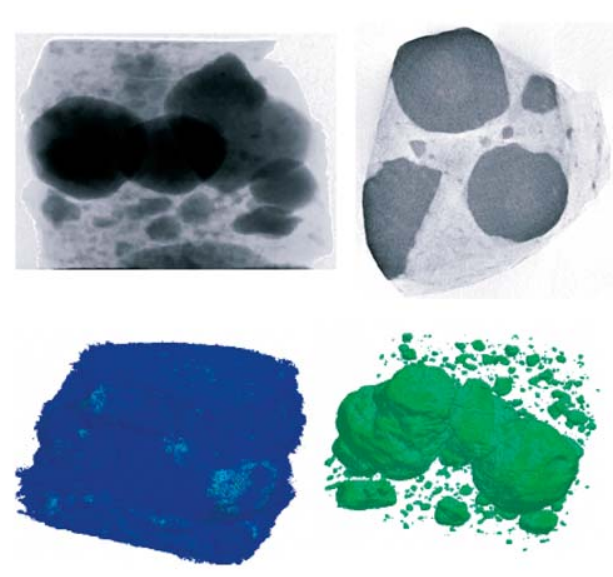

(b)

Figure 1.XMT scans of dispersible aspirin tablets (Boots, B.P. $75 \mathrm{mg}$ ). From left to right and top to bottom are X-ray image, tomogram, and 3D views of the sample and spatial distribution of a component for (a) a whole tablet and (b) a quarter of a tablet. Darker pixels in the X-ray images and tomograms indicate denser materials.

across) and well separated (i.e., by at least one pixel on all sides), shapes of individual particles can be obtained. Of course, the sample can be a single particle or granule, and the shape as well as internal structure can be obtained. X-ray tomography therefore provides a non-invasive and nondestructive means to obtain 3D information of a given sample, be it a human body, a tablet, a single particle, or a piece of other materials.

XMT works on the same principles, but with a much higher spatial resolution, typically a few microns per pixel for micro-focus CT scanners and a sub-micron pixel resolution for nano-focus CT scanners that are now becoming commercially available. Accompanying the high resolution is a restriction on sample size, typically a few to a few tens of millimetres. The actual pixel resolution may be calculated by dividing the sample size by the number of detectors used for collecting the X-ray images. For instance, in SkyScan 1072 , the CCD sensor array is $1024 \times 1024$; a 4 -mm sample can be scanned at 4- $\mu \mathrm{m} /$ pixel resolution.

Figure 1 shows two examples of aspirin tablets scanned at different resolutions. The whole tablet, measuring $7 \mathrm{~mm}$ across and $3 \mathrm{~mm}$ high, was scanned at $10-\mu \mathrm{m} /$ pixel resolution. A quarter of another tablet was scanned at $6-\mu \mathrm{m} /$ pixel resolution. Although a single X-ray image is usually enough to qualitatively assess if the components are uniformly distributed within the tablet, a full reconstruction of the structure is required to analyse quantitatively the distributions or to predict numerically their properties such as dissolution.

\section{Modelling Structures of Granules and Tablets}

At present, $\mathrm{XMT}$ is probably the only non-destructive technique for obtaining full 3D structural information of samples. However, the technique requires that physical samples be made available in advance, and in this sense, it is mostly a tool for'post-mortem' analyses. A computational model, on the other hand, can be a more cost effective tool for product formulation and design, as it can help either to understand retrospectively the observed behaviour of a finished product or to predict the likely behaviour or properties of a new product before it is actually produced. DigiPac is one such software model. It has the two aforementioned capabilities because it provides a quantitative link between properties of individual particles in the feedstock and properties of an assembly of these particles, that is, the product.

DigiPac (4) takes digitised real particle shapes as input to predict packing structures. In DigiPac, both the packing space (or container) and the particles are mapped in a lattice grid, and particles move one grid cell at a time following a pre-defined set of rules that, in principle, can be governed by physical laws or simply a random motion plus a tendency to move in a particular direction (e.g., downwards in the case of particle settling under gravity). The use of a lattice approach simplifies collision and overlap detection between particles of complex shapes, making it easier to pack particles of any sizes and shapes in a container of arbitrary geometry. Once digitised, complex shapes are no more difficult to handle than spheres in generating a packing structure using the DigiPac approach.

There are several ways to digitise particles: converting from CAD data for designed objects; directly generating objects using computer software for mathematically describable simple shapes such as ellipsoids, cylinders, and crystals; reconstructing from 2D SEM or photo images using customized software routines; and 3D optical or X-ray scanning of particles. Figure 2 contains some examples of XMT digitised powder particles ranging in size from 10 to several hundred microns. For confidentiality, identities of the powders are withheld. They are simply referred to as PWD1 to PWD8. An example of packing structure for PWD3 is given in Figure 2b. Predicted and measured packing densities are compared for eight different powders in Figure 2c. Up to 175 individual particles from each of the eight powders were sampled and scanned using X-ray microto- 


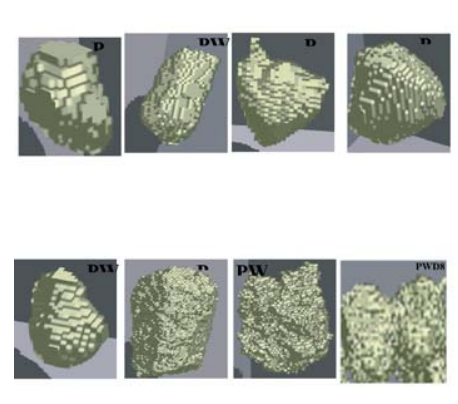

(a)

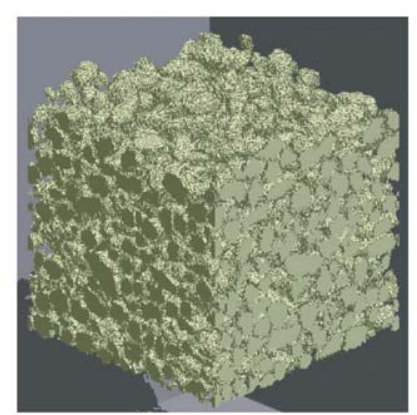

(b)

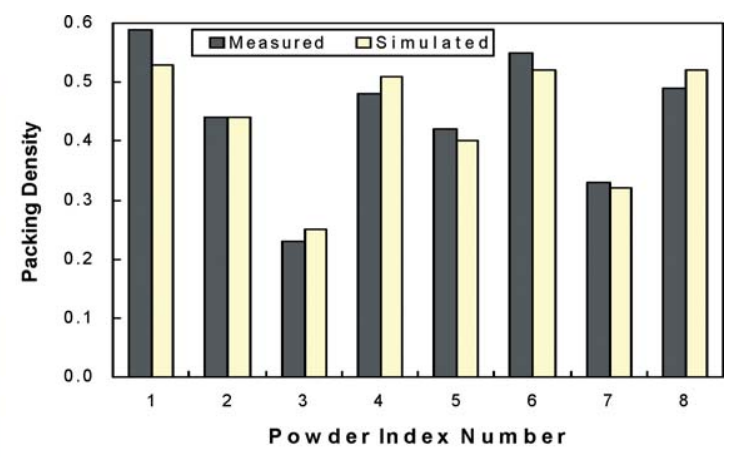

(c)

Figure 2. (a) Examples of digitised particles, one from each powder. (b) An example of DigiPac generated packing structures (PDW2). (c) Comparison between predicted and measured packing density for each powder.

mography to obtain their shapes and internal porosities. Of the eight types of powder particles, some (PWD1, PWD4, PWD6, and PWD8) are crystalline, and some (PWD3 and PWD7) are agglomerates of various internal porosities. The scanned particles are deemed to be representative of size and shape distributions for each powder and duplicated many times to make up the feedstock (containing several thousand particles) for each packing simulation. Periodical boundary conditions are used to minimise wall effects. For all the simulations, the standard deviation of predicted bulk packing density is less than 0.002 . Further details of this case study and other real-life examples can be found in $(5,6)$.

Achieving uniform blending is a recurring problem in the production of solid form drugs. Uniformity also affects the dissolution rate of the drug. It is well known that particles of different sizes tend to segregate as the powder is poured into a container and/or if the container is vibrated or shaken. However, the part played by particle shape in segregation is less extensively studied. For computer modelling and simulation, conventional packing models cannot easily handle a large number of different shapes. With DigiPac, segregation of particles of different shapes (and sizes) can be easily simulated. Two illustrative examples of particle segregation are given in Figure 3. In the first example, Figure 3a, particles differing both in size and shape were poured into a cylindrical container that was subsequently shaken a number of times. The second example illustrates that particles of the same size but different shape can still segregate during the powder-handling process. In Figure 3b, a binary mixture of particles of two different shapes was packed to form an initial packing. Periodical boundary conditions were used on the sides to eliminate the so-called wall effect, allowing a small-scale simulated sample to represent the bulk. The packing was subsequently shaken 100 times to produce the segregated structure in Figure $3 \mathrm{c}$. It is interesting to note that particles of the same shape tend to form clusters in the initial packing structure (Figure 3b), which appears to be uniform at a macroscopic level. Obviously, the number of particles used in the examples is far less than in any real process; however the number of shakes required to produce a predefined degree of segregation can be a useful indicator of the relative tendency for segregation of a given mixture.

\section{Modelling Flow Through Granules and Tablet Structures}

Lattice Boltzmann Method (LBM) is a digital equivalent of the convectional computational fluid dynamics (CFD). While CFD solves numerically the Navier-Stokes equation, the governing equation of fluid flow, directly, LBM, which has its root in the kinetic theory for gas, treats fluid as imaginary'particles' of varying density, residing in a regular grid and only interacting with their immediate neighbours in the grid. Being a mesoscale model, LBM does not provide a microscopically precise picture of a real fluid flow, but it has been shown that macroscopically, the predicted flow behaviour, as described by Navier-Stokes, can be recovered under certain conditions (e.g., low Mach number). This lattice approach and data localisation makes it adept at dealing with complex geometries such as those of porous media. It is a natural choice for the digital structures obtained either by DigiPac or from XMT scans.

While a fuller treatment of the subject can be found in the literature elsewhere $(7,8)$, only the basic features of LBM are presented here. The LBM maps physical space onto a regular lattice grid. Associated with each node is a set of mass probability distributions, also referred to as particle distribution functions or simply density. These distributions propagate to neighbouring nodes as part of mass and momentum transfer, along fixed velocity vectors at each time step, followed by simple collisions designed to conserve mass and momentum. The collision operator is chosen such that the particle velocity distributions at each node obey the Navier-Stokes equations in the low Knudsen number and low Mach number regime. In the LBM, the evolution of the particle distribution functions is described as: 
$f_{i}\left(\vec{x}+\vec{e}_{i}, t+1\right)=f_{i}(\vec{x}, t)-\frac{1}{\tau}\left[f_{i}(\vec{x}, t)-f_{i}^{e q}(\vec{x}, t)\right]$

$f_{0}^{e q}=\frac{\rho}{3}\left[1-\frac{3}{2} u^{2}\right]$

$f_{i}^{\text {eq }}=\frac{\rho}{18}\left[1-3\left(\vec{e}_{i} \cdot \vec{u}\right)+\frac{9}{2}\left(\vec{e}_{i} \cdot \vec{u}\right)^{2}-\frac{3}{2} u^{2}\right]$ in 6 orthogonal directions

$f_{i}^{e q}=\frac{\rho}{36}\left[1-3\left(\vec{e}_{i} \cdot \vec{u}\right)+\frac{9}{2}\left(\vec{e}_{i} \cdot \vec{u}\right)^{2}-\frac{3}{2} u^{2}\right]$ in 12 diagonal directions

where $\vec{x}$ is the $(x, y, z)$ coordinates of node $i, \overrightarrow{\mathrm{e}}$ the $i$-th component of the velocity vector $u, t$ the time, $f_{i}^{\text {eq }}$ the equilibrium distribution, and $\tau$ a numerical constant related to fluid viscosity. The left hand side of the equation describes the propagation or translation, whereas the right hand side describes the collision operation in which all the instantaneous distribution functions relax to their equilibrium values at a single rate set by $\tau$. The time step in an LBM simulation is customarily set to 1 , as is the magnitude of the velocity components. As such, everything else (e.g., length scales, velocities, and pressure) is in lattice units. The results can be rescaled to physical units, if necessary. The so-called D3Q19 scheme is adopted here for calculating the equilibrium distributions. This scheme operates on a $3 \mathrm{D}$ cubic lattice grid with each grid cell interacting with 18 (out of 26) of its closest neighbours.

Figure 4 shows example flow distributions calculated using LBM through a packing structure generated by DigiPac and a foam structure imaged using XMT. A further example of the application of LBM to flow through flocs and filter cakes in a filtration setup can be found elsewhere (9).

\section{Simulation of Dissolution}

Drug dissolution is an important issue for the pharmaceutical industry. The usual approach currently taken by the industry is essentially an empirical one (1). Theoretical models do exist (10-14), but most only deal with regular geometries. An exception is a recent work by Stepaneck (15), who modelled small granules consisting of irregular particles and a binder. The model is based on a vector approach, using a surface mesh to represent a particle geometry and conventional CFD to calculate flow.

DigiPac takes a different-the digital-approach, which has the advantage of easy and direct incorporation of real particle shapes as they are measured (by XMT). The structure, either simulated using DigiPac or acquired using XMT, is described in a lattice grid that is also used by the LBM simulation to calculate the flow field; with the finite difference method, the same grid can be used to solve the governing equations for dissolution:

$$
\begin{gathered}
\frac{\partial C}{\partial t}+\left(u_{x} \frac{\partial C}{\partial x}+u_{y} \frac{\partial C}{\partial y}+u_{z} \frac{\partial C}{\partial z}\right)=D\left(\frac{\partial^{2} C}{\partial x^{2}}+\frac{\partial^{2} C}{\partial y^{2}}+\frac{\partial^{2} C}{\partial z^{2}}\right)+Q \\
\frac{\partial W}{\partial t}=-k S\left(C_{\text {sat }}-C\right)
\end{gathered}
$$

(3)

Equation (2) is the convection-diffusion equation that describes the concentration distribution in a liquid. $C$ is mass concentration $\left[\mathrm{kg} \mathrm{m}^{-3}\right], t$ the time $[\mathrm{s}],\left(u_{x}, u_{y}, u_{z}\right)$ fluid velocity components $\left[\mathrm{m} \mathrm{s}^{-1}\right], D$ diffusion constant $\left[\mathrm{m}^{2} \mathrm{~s}^{-1}\right]$, and $(x, y, z)$

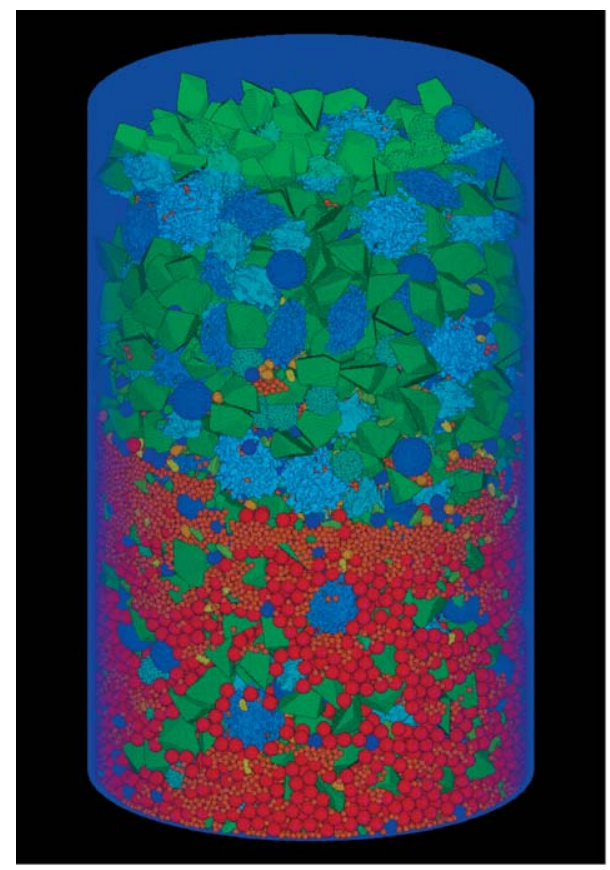

(a)

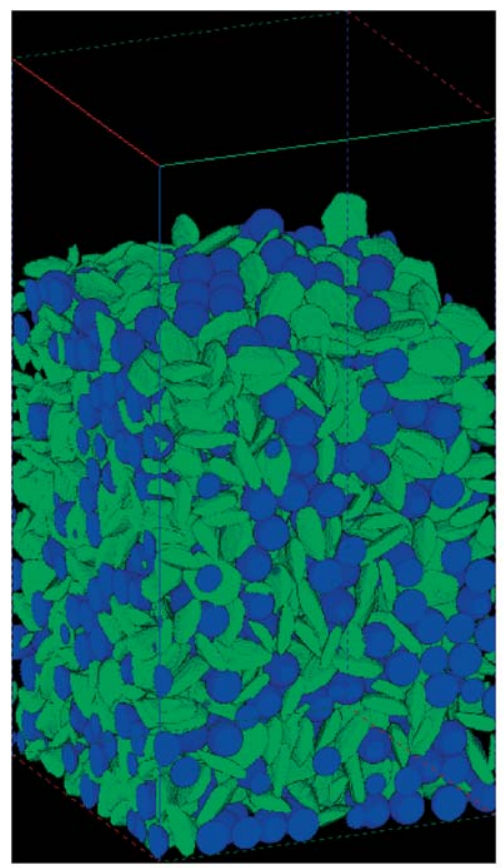

(b)

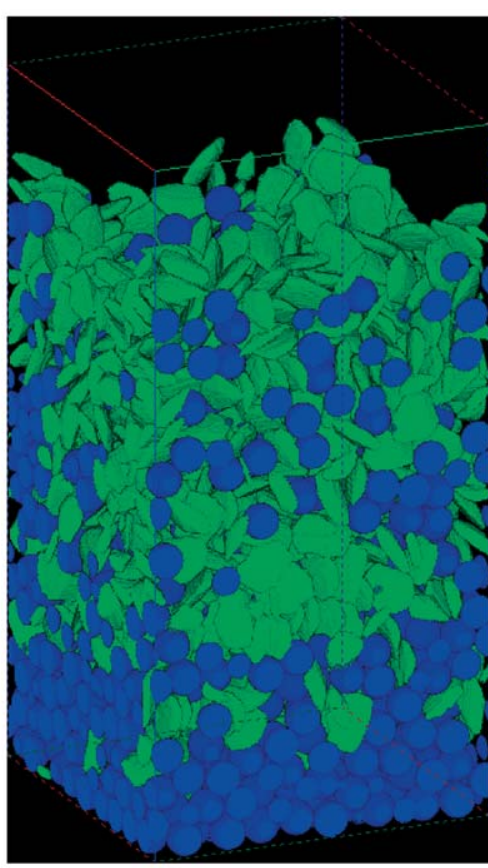

(c)

Figure 3. Examples of particle segregation. (a) Particles of different sizes and shapes. (b) and (c) Particles of different shapes but same equivalent volume size. Pseudo colours are used to identify particles of different sizes and shapes. 

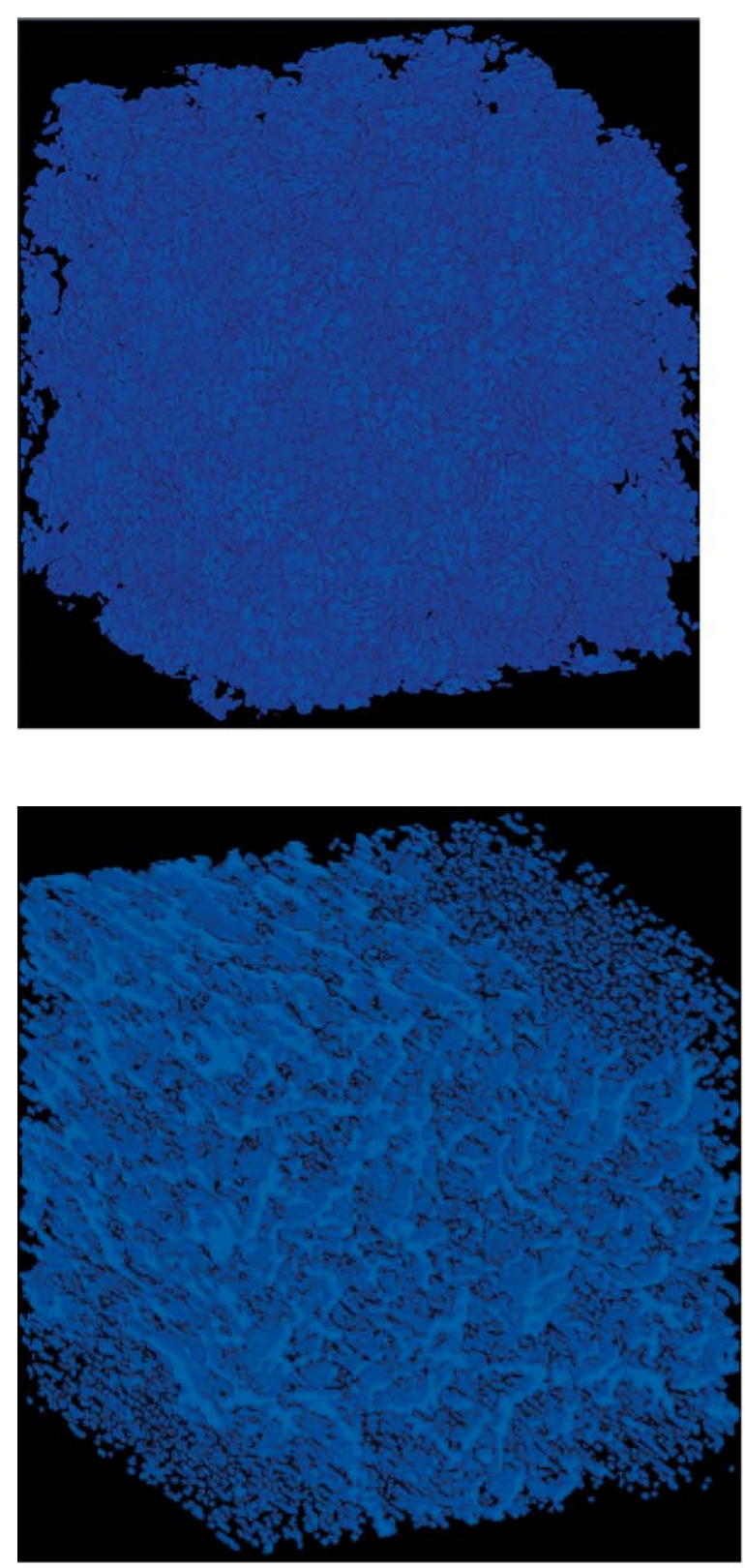

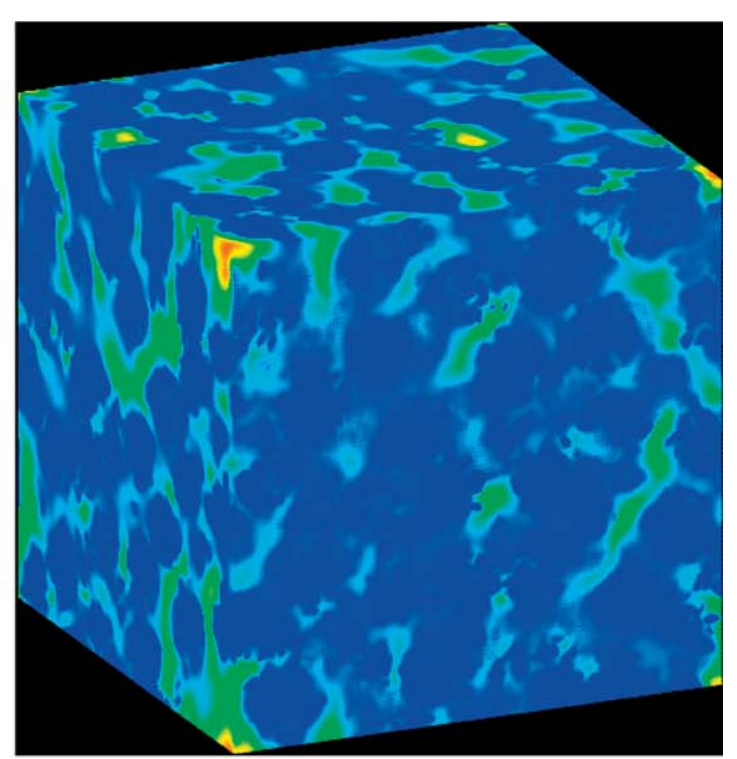

(a)

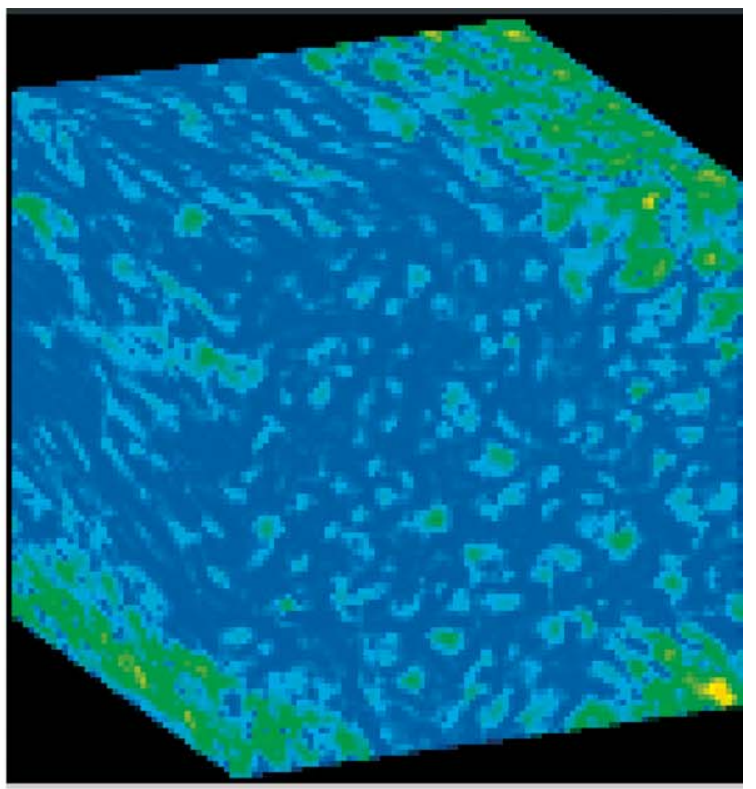

(b)

Figure 4. Examples of LBM simulated flow distributions through porous media. (a) Packing structure of agglomerates generated by DigiPac and flow distribution. (b) Matrix structure of a piece of foam imaged using XMT and flow distribution. A red to blue colour spectrum is used to indicate flow velocity, with red being high and blue low.

Cartesian coordinates [m]. Depending which terms are retained in Equation (2), four different scenarios can be calculated:transient or steady state with or without convection. It is assumed that at the solid/liquid interface, dissolution can be described as a first-order reaction, Equation (3), which, for drug dissolution, is more commonly known as the Noyes-Whitney equation. In Equation (3), W is the weight of the drug dissolved $[\mathrm{kg}], k$ dissolution constant $\left[\mathrm{m} \mathrm{s}^{-1}\right], S$ surface area $\left[\mathrm{m}^{2}\right], C_{\text {sat }}$ solubility or saturation concentration of the drug $\left[\mathrm{kg} \mathrm{m}^{-3}\right]$, and $C$ bulk concentration in the liquid phase at the interface $\left[\mathrm{kg} \mathrm{m}^{-3}\right]$. The source term, $Q\left[\mathrm{~kg} \mathrm{~m}^{-3} \mathrm{~s}\right]$ in Equation (2), provides the link between the two equations at the interface between a dissolving solid phase and the solvent phase.

To most drug testing practitioners, the release profile is of the most interest. This can be obtained by solving Equation (2) in the transient state, that is, retaining the time-dependent term and following through the evolution of concentration distribution over time. To save simulation time, a short-cut approach may also be employed. In this approach, the steady state concentration distribution is calculated according to Equation (2) without the time-dependent term. The concentration is then used in Equation (3) to estimate the time required for each of the exposed solid pixels 

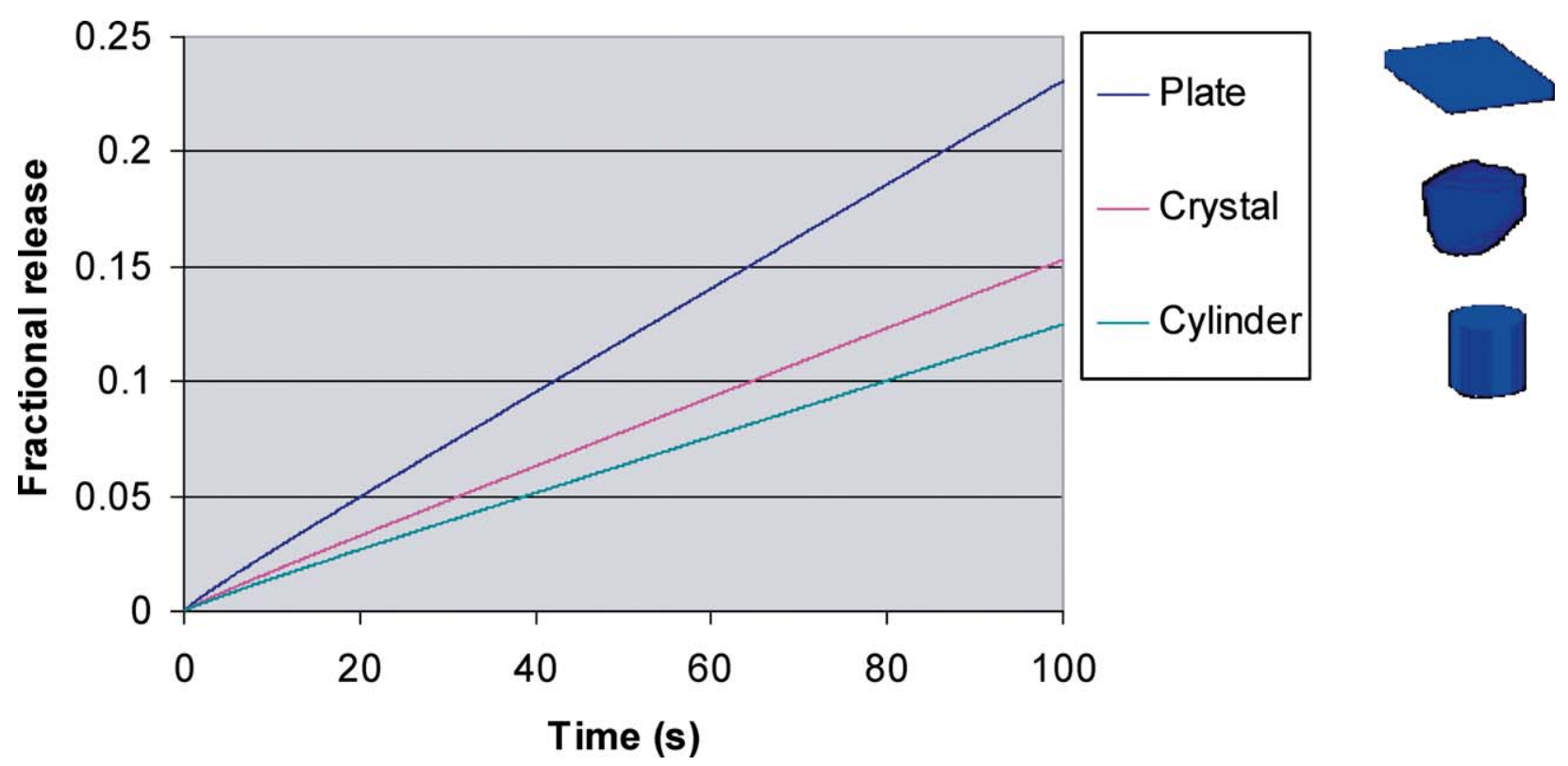

Figure 5. Simulation results showing three particles having the same surface area $\left(2235 \mu \mathrm{m}^{2}\right)$ but different dissolving behaviour. Mass density (1250 $\left.\mathrm{kg} / \mathrm{m}^{3}\right)$, diffusion constant $\left(1.0 \times 10^{-10} \mathrm{~m}^{2} \mathrm{~s}^{-1}\right)$, and dissolution coefficient $\left(1.0 \times 10^{-4} \mathrm{~m} \mathrm{~s}^{-1}\right)$.

to completely dissolve. A solid pixel is deemed to have completely dissolved if the remaining density (or mass concentration) of the pixel drops below the value of saturation concentration, and thereafter the pixel is treated as part of the liquid phase. Both concentration distribution and velocity fields are updated at specifiable intervals. The intervals can be specified based either on the time (e.g., every second or minute) or the number of solid pixels or layers dissolved (e.g., for every 100 pixels dissolved or every layer of solid pixels dissolved). Provided this interval is small enough, the short-cut approach should produce an estimated release profile very close to that obtained using the more rigorous approach, while significantly saving the computing time. The release profiles shown in this article were produced using this short-cut approach.

The dissolution model can be applied at several different length scales - the whole tablet, individual granules, or individual fine particles - to assess how shape, internal (porous) structure, and the presence of obstacles affect the overall dissolution rate with or without a superimposed flow field. The input microstructure may contain any number of different components; the governing equations are solved for each individual component. For each grid cell, the discretised form of the above equations is such that both $k$ and $D$ can be variables of time and space. This allows interactions between the dissolving components and their effects on subsequent dissolution of individual components to be taken into account, if such interactions can be predefined. For the sake of convenience, however, they were assumed to be a constant for each component in the simulation examples given below.

At first sight, the Noyes-Whitney equation seems to suggest that for the same substance, the dissolution rate is only dependent on the exposed surface area. Figure 5 shows that particle shape can also play a role. The three particles have identical surface area to start with, but they dissolve differently, according to the model. This is because the spatial distribution of the exposed surface area has an effect on the precise value of $C$ in Equation (3). In other words, the rate at which the dissolved material is carried away from the dissolving surface by diffusion (and convection) also influences the observed dissolution rate. This effect is often ignored by practitioners of dissolution testing, but it can be significant, especially if the process is diffusion limited.

Figure 6a shows that particles of the same material and size can dissolve at different rates if they have different shapes. For granules, the dissolution rate is also dependent on spatial distribution of constituent particles. So in this example, although Component 2 dissolves faster than Component 1 if standalone, in a granule setup, it may actually be released at a slower rate. It can be seen from Figure $6 \mathrm{~b}$ that the overall dissolution rate can be enhanced by convection, but the relative rate of release for each individual component remains the same and is controlled by the microstructure of the granule. In all these simulations, the primary particles had an equivalent volume size of 12 microns. The agglomerate, consisting of 20 of these primary particles, was generated using DigiPac. Both components were given identical mass density $\left(1250 \mathrm{~kg} \mathrm{~m}^{-3}\right)$, diffusion constant $\left(1.0 \times 10^{-10} \mathrm{~m}^{2} \mathrm{~s}^{-1}\right)$, and dissolution coefficient $(1.0 \times$ $\left.10^{-4} \mathrm{~m} \mathrm{~s}^{-1}\right)$. In the convection-diffusion case, Figure $6 \mathrm{~b}$, the LBM calculated flow field, the maximum velocity was $1 \times 10^{-4}$ $\mathrm{m} \mathrm{s}^{-1}$.

It is possible to use the scanned tablet 'as is' in the simulation. However, as DigiPac does not yet simulate the disintegration of the tablet into small dispersed granules, it would not be of practical interest if we did. Instead, in the last example, Figure 7, the XMT-scanned quarter part of an 


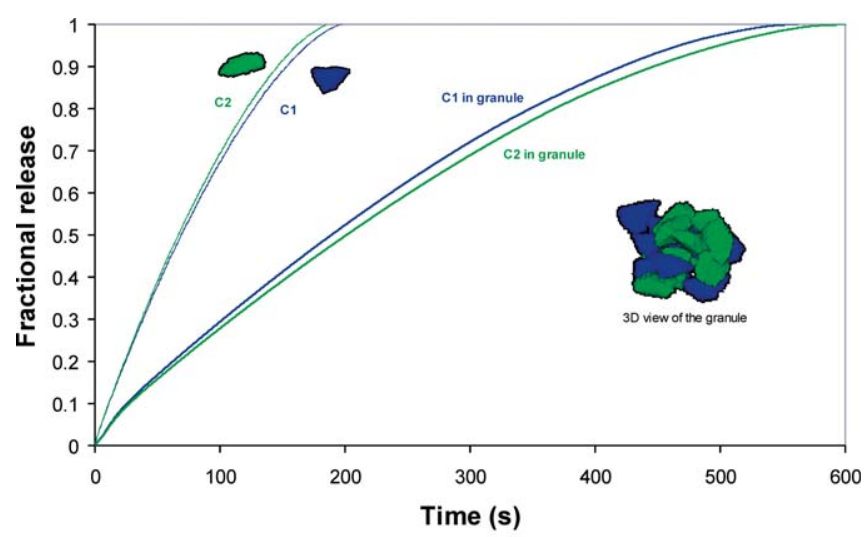

(a)

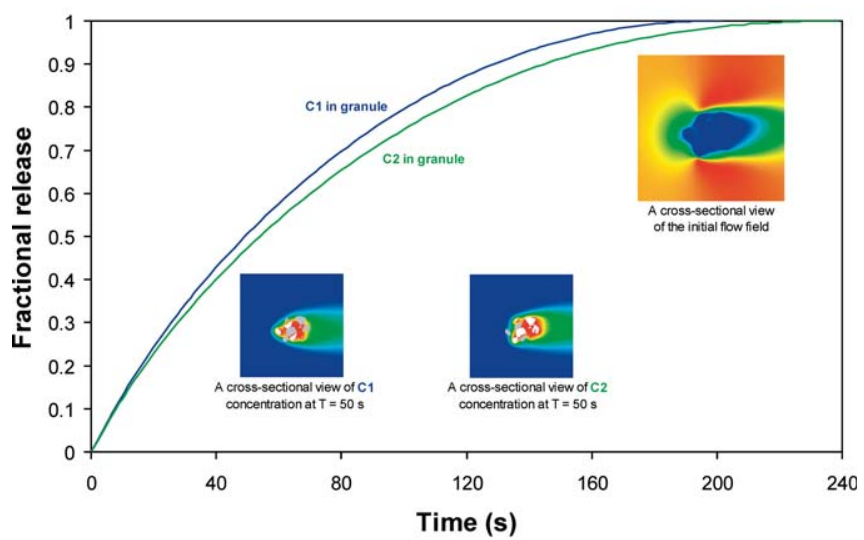

(b)

Figure 6. Simulated dissolution profiles of (a) standalone component particles and their agglomerate under diffusion-only conditions and (b) the constituent components of the same agglomerate under flow conditions.

aspirin tablet was rescaled from $3.2 \mathrm{~mm}$ down to $0.4 \mathrm{~mm}$ and used to mimic a binary granule containing $35 \% \mathrm{v} / \mathrm{v}$ of a slow-dissolving incipient $\left(k=1.0 \times 10^{-6} \mathrm{~m} \mathrm{~s}^{-1}, \rho=1000 \mathrm{~kg}\right.$ $\mathrm{m}^{-3}$ ) embedded in a low density, fast dissolving binder material $\left(k=2.0 \times 10^{-5} \mathrm{~m} \mathrm{~s}^{-1}, \rho=800 \mathrm{~kg} \mathrm{~m}^{-3}\right)$. Diffusion coefficient was set to $2.0 \times 10^{-10} \mathrm{~m}^{2} \mathrm{~s}^{-1}$. In this hypothetical example, the fast dissolving binder material, which made up nearly $2 / 3$ of the total volume of the granule, had a dissolution constant 20 times that of the incipient. Figure $7 \mathrm{~b}$ shows crosssectional views of the granular structure and concentration distribution of each component after 6 hours and 40 minutes of dissolution, during which time about $90 \%$ of the binder material and $50 \%$ of the incipient dissolved. White pixels indicate the spatial distribution of the solid component for which the concentration map is displayed; and grey pixels belong to other solid components. The green curve in Figure $7 \mathrm{c}$ for the binder material is typical of a non-disintegrating tablet, since the binder is essentially an amorphous solid mass whose exposed surface area gradually decreases during dissolution. The red curve for the incipient resembles a typical profile for a disintegrating tablet. This is as

\footnotetext{
${ }^{2}$ DigiPac $^{T M}$ is a product from Structure Vision www.structurevision.com.
}

expected since as the binder dissolves away, the incipient gradually becomes separate individual particles. However, as the inter-particle separation distances were small and did not change during the simulation, the inflection is not as obvious as it would have been if the particles had been allowed to separate more as per real disintegration.

\section{Conclusions}

By making full use of 3D shape information, the digital approach has the potential to be a valuable tool for process engineers in the pharmaceutical industry to better understand the effects of particle size and shape on the packing structure and structure-related properties such as dissolution. Further developments in these areas can be anticipated (e.g., tablet pressing, swelling, disintegration, and deaggregation).

The time and cost of getting a drug to market has long been a critical success factor for pharmaceutical companies. For a new drug or a new formulation, the current empirical approach is a significant element of this time and cost and, by its nature, involves overhead, for example:

- The drug must physically exist; therefore it must be produced at every stage that requires testing and retesting.

- The results of dissolution tests require additional time to examine and explain observations.

- Previous empirical data may be of little use, even for a minor change to formulation.

By using modules from DigiPac ${ }^{2}$, a state-of-the-art mesoscale software suite, a number of dissolution simulations have been performed. The dissolution simulations take into account the effects of the actual shape of the particles or granules (not a mathematical approximation), their compaction and packing, and together with a number of other simulations, provide a library of "what ifs" without the drug actually existing in a physical state. The results and observations from DigiPac are explicit, graphical (nano level, if required), and can be loaded into another application. While the simulations shown in this paper are only a small part of DigiPac's capabilities, the example scenarios described could help significantly reduce the time and cost to market for new or reformulated drugs.

\section{References}

1. Hanson, R.; Gray, V. Handbook of Dissolution Testing, $3^{\text {rd }}$ ed.; Dissolution Technologies, Inc.: Hockessin, DE, 2004.

2. Jia,X.;Williams, R. A. The shape of things to come. Pharm.Tech.Eur, in press.

3. Kak, A. C.; Slaney, M. Principles of Computerized Tomographic Imaging. Society for Industrial and Applied Mathematics:Philadelphia, 2001. Also available electronically at http://www.slaney.org/pct/pct-toc.html.

4. Jia, X.;Williams, R. A. A packing algorithm for particles of arbitrary shapes. Powder Technol. 2001, 120, 175-186. Also, Jia, X.; Williams, R. A. Object interaction simulation. U.K. Pat. Appl. P888399B, 2001.

5. Gan, M.;Gopinathan, N.; Jia, X.;Williams, R. A. Predicting 


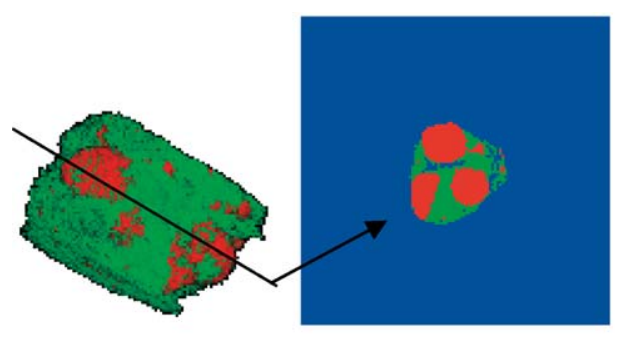

(a)
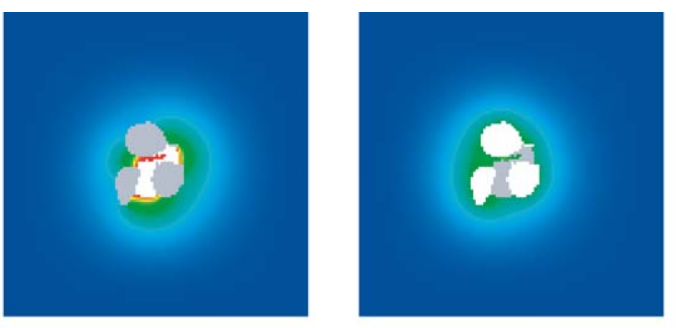

(b)

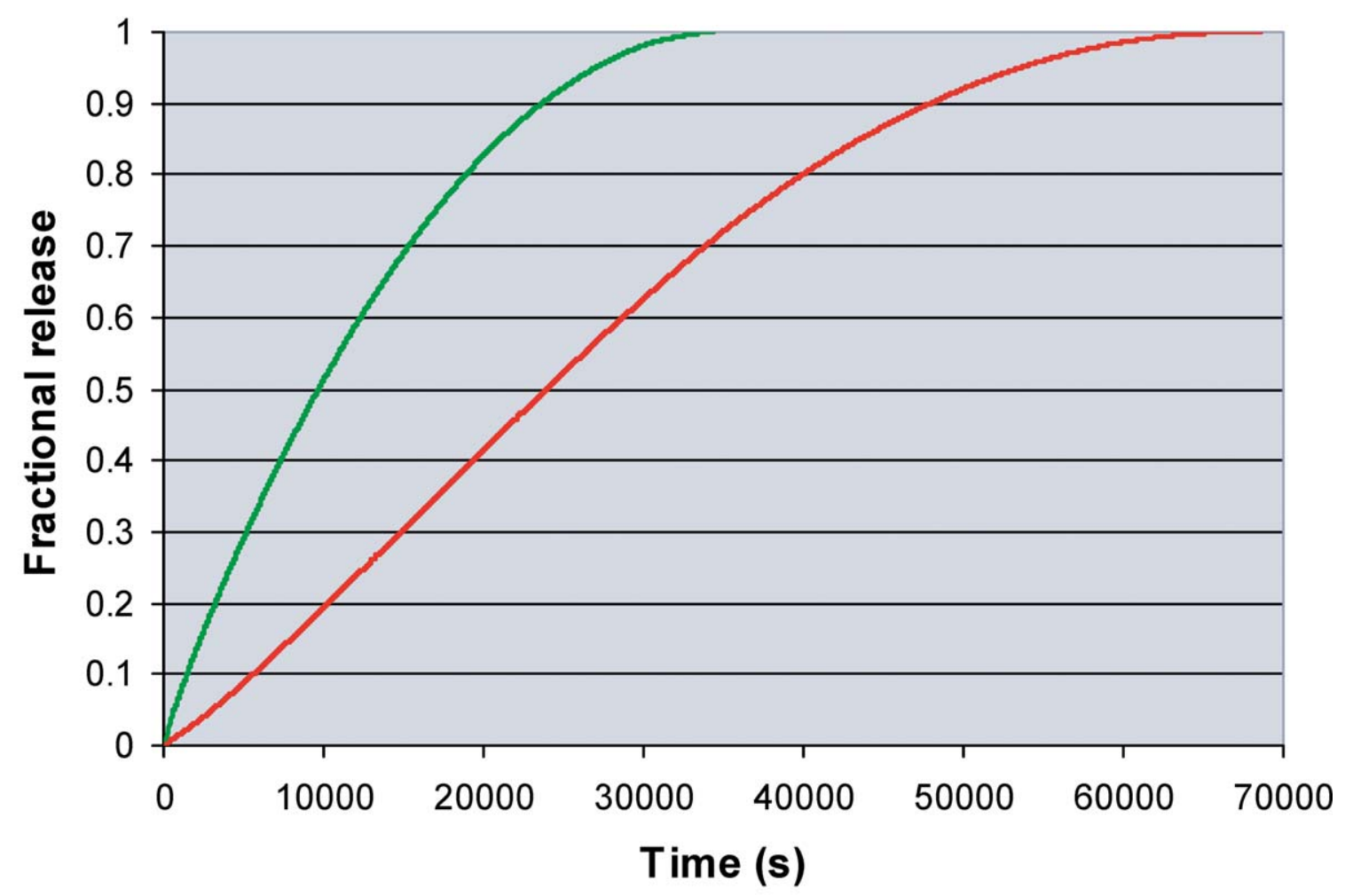

(c)

Figure 7. Simulated dissolution of a granule with slow-dissolving incipient particles embedded in a fast-dissolving binder material. (a) 3D view and a crosssectional view of the initial granular structure. (b) Cross-sectional views of the granular structure and concentration distribution of each component in the vicinity of the granule at $t=24000 \mathrm{~s} ; 90 \%$ of binder and $50 \%$ of incipient have dissolved. (c) Release profiles for excipient and incipient.

packing characteristics of particles of arbitrary shapes. KONA Powder and Particle 2004,22,82-93.

6. Jia, X.; Gan, M.; Williams, R. A.; Rhodes, D. Validation of a digital packing algorithm in predicting powder packing densities. Presented at Particulate Systems Analysis 2005, Stratford on Avon, U.K., September 2005.

7. Wolf-Gladrow, D. A. Lattice-Gas Cellular Automata and Lattice Boltzmann Models: An Introduction (Lecture Notes in Mathematics). Springer-Verlag:Berlin Heidelberg, 2000.

8. Succi, S. The Lattice Boltzmann Equation: For Fluid Dynamics and Beyond. Oxford University Press: Oxford, UK, 2001.
9. Selomulya, C.; Jia,X.;Williams, R. A. Direct prediction of structure and permeability of flocculated structures and sediments using 3D tomographic imaging. Chem. Eng.Res.Des. 2005, 83 (A7),844-852.

10. Bekri, S.;Thovert, J. F.;Adler, P. M. Dissolution of porous media. Chem. Eng.Sci. 1995, 50, 2765-2791.

11. Dokoumetzidis, A.; Kosmidis, K.; Argyrakis, P.; Macheras, P. Modeling and Monte Carlo simulations in oral drug absorption. Basic Clin. Pharmacol.Toxicol.2005, 96, 200-205.

12. Wu, N.;Wang, L. S.;Tan, D. C. W.;Moochhala, S. M.;Yang,Y.Y. Mathematical modeling and in vitro study of controlled drug release via a highly swellable and dissoluble 
polymer matrix: polyethylene oxide with high molecular weights. J. Controlled Release 2005, 102,569-581.

13. Lansky, P.; Lanska, V.;Weiss, M. A stochastic differential equation model for drug dissolution and its parameters. J. Controlled Release 2004, 100, 267-274.

14. Sarisuta, N.; Jateleela, S.;Tourtip, T. Dissolution kinetics of three-component compressed solid mixtures with largely different solubilities:Flaking spheres. J. Pharm. Sci. 2000,89 (9), 1196-1211.

15. Stepanek, F. Computer-aided product design - granule dissolution. Chem. Eng. Res.Des. 2004, 82 (A11), 1458-1466. 